\title{
High serum osteopontin levels are associated with prevalent fractures and worse lipid profile in post-menopausal women with type 2 diabetes
}

\author{
T. Filardi ${ }^{1}$ V. Carnevale ${ }^{2} \cdot$ R. Massoud ${ }^{3} \cdot$ C. Russo ${ }^{3} \cdot$ L. Nieddu $^{4} \cdot$ F. Tavaglione $^{1} \cdot$ I. Turinese $^{5} \cdot$ A. Lenzi ${ }^{1} \cdot$ E. Romagnoli $^{1}$. \\ S. Morano ${ }^{1}$ (1)
}

Received: 7 March 2018 / Accepted: 13 June 2018

(C) Italian Society of Endocrinology (SIE) 2018

\begin{abstract}
Purpose Patients with type 2 diabetes (T2DM) have increased fracture risk. Osteopontin (OPN) is a protein involved in bone remodeling and inflammation. The aim of this study was to evaluate the association of OPN with fracture prevalence and with metabolic parameters in post-menopausal women with T2DM.

Methods Sixty-four post-menopausal women with T2DM (age 67.0 \pm 7.8 years, diabetes duration $8.9 \pm 6.7$ years), enrolled in a previous study, were followed up (3.6 \pm 0.9 years). Previous fragility fractures were recorded. The FRAX score (without BMD) was calculated and biochemical parameters (plasma glucose, HbA1c, lipid profile and renal function) were assessed. Serum 25OH-vitamin D, calcium, PTH and OPN were evaluated at baseline. The association between OPN and fracture prevalence at baseline was evaluated by a logistic model.

Results OPN levels were higher in patients with previous fractures (n.25) than in patients without previous fractures at baseline (n.39) ( $p=0.006)$. The odds of having fractures at baseline increased by $6.7(1.9-31.4,95 \% \mathrm{CI}, p=0.007)$ for each increase of $1 \mathrm{ng} / \mathrm{ml}$ in OPN levels, after adjustment for vitamin D and HbA1c levels. Fracture incidence was 4.7\%. Higher OPN associated with a decrease in HDL-cholesterol $(p=0.048)$, after adjustment for age, basal HDL-cholesterol, basal and follow-up HbA1c and follow-up duration. 25OH-vitamin D associated with an increase in FRAX-estimated probability of hip fracture at follow-up ( $p=0.029)$, after adjustment for age, $25 \mathrm{OH}$-vitamin $\mathrm{D}$ and time.

Conclusions In post-menopausal women with T2DM, OPN might be a useful marker of fracture and worse lipid profile.
\end{abstract}

Keywords Osteopontin · Type 2 diabetes $\cdot$ Fracture risk $\cdot$ Lipid profile $\cdot$ FRAX tool $\cdot$ 25OH Vitamin D

S. Morano

susanna.morano@uniroma1.it

1 Department of Experimental Medicine, Policlinico Umberto I, "Sapienza" University of Rome, Viale del Policlinico 155, 00161 Rome, Italy

2 Unit of Internal Medicine, Casa Sollievo della Sofferenza Hospital, IRCCS, San Giovanni Rotondo, Italy

3 Department of Experimental Medicine and Surgery, Tor Vergata University, Rome, Italy

4 Faculty of Economics, UNINT University, Rome, Italy

5 Department of Public Health and Infectious Diseases, "Sapienza" University, Rome, Italy

\section{Introduction}

Type 2 diabetes mellitus (T2DM) is a chronic disease with increasing global prevalence. It can lead to macro- and microvascular complications, causing marked disability. It has also been demonstrated that patients with T2DM have higher fracture risk, compared with subjects without diabetes [1-5]. Paradoxically, bone mineral density (BMD) is normal or even increased in most men and women with T2DM. Thus, the clinical utility of BMD in these patients has been questioned $[2,3]$. On the other hand, the algorithms based on clinical risk factors, such as the most widespread FRAX algorithm [6], underestimate fracture risk in the high-risk population of patients with T2DM [7, 8]. These concern about the predictive role of the most commonly used diagnostic tools, stimulates to search for possible alternative markers of skeletal fragility in T2DM. Osteopontin (OPN) 
is a non-collagenous matrix protein expressed in bone tissue, which is involved in bone remodelling and capable to activate bone resorption $[9,10]$. In addition, OPN is a protein with pleiotropic physiological functions, involved in both acute and chronic inflammation [11] and there is increasing evidence about its role as a novel biomarker of cardiovascular risk in patients with T2DM [12].

The aim of this study was to evaluate the association between OPN and fracture prevalence, in a sample of postmenopausal women with T2DM. Considering the possible role of OPN as a cardiovascular risk biomarker, in these patients we also longitudinally investigated the association between OPN circulating levels and some glyco-metabolic parameters.

\section{Methods}

Sixty-four post-menopausal women with T2DM (mean age $67.0 \pm 7.8$ years, mean diabetes duration $8.9 \pm 6.7$ years), enrolled in a previous larger cross-sectional multicenter study [8], were followed up longitudinally in the outpatient clinics of Policlinico Umberto I, "Sapienza" University Hospital of Rome. The previous multicenter study had recruited n.974 patients with T2DM and n.777 control subjects in three Italian outpatient clinics and was aimed to define whether distinct clinical profiles of patients with T2DM were associated with higher FRAX values. A sub-group of patients was selected for follow up, through the following inclusion criteria: female sex; T2DM; post-menopausal status; age $<85$ years. Exclusion criteria were as follows: reported diagnosis or pharmacologic therapy for osteoporosis; all clinical conditions associated with secondary osteoporosis (endocrine, haematological, gastrointestinal, rheumatic and renal diseases); treatment with thiazolidinediones, methotrexate, antiepileptic agents, glucocorticoids, heparin and orlistat; drug and alcohol abuse; psychiatric diseases; malignant neoplasms. All patients gave written informed consent, the protocol was approved by the hospital ethics committee and was performed in accordance with the ethical standards laid down in the 1964 Declaration of Helsinki and its later amendments.

Both at enrolment and at follow-up visits a detailed medical history, including information about UV exposure [13], the recording of previous/incident fractures and major cardiovascular events, a complete medical examination were performed and all medications were recorded. The FRAX scores (based on anthropometric data, without BMD measurement) were calculated at baseline and at final visit [6]. All patients were also screened for the presence of T2DM complications (cardiovascular disease, diabetic retinopathy and neuropathy). Both at baseline and follow up, the following biochemical parameters were assessed: fasting plasma glucose, HbA1c, lipid profile and renal function markers. GFR was estimated by the CKD-EPI equation [14]. Circulating levels of $25 \mathrm{OH}$-vitamin D, calcium, PTH and OPN were evaluated at baseline only. At follow up, all patients were asked about incident clinical fragility fractures from baseline.

General chemistry parameters were assessed by an automated multi-analyzer (Dimension Vista, Siemens technology, USA). HbA1c was measured by HPLC (Menarini Diagnostics, Milan, Italy). The levels of $25 \mathrm{OH}$-vitamin D and PTH were measured by an automated chemiluminescence immunoassay (ADVIA Centaur XP, Siemens technology, USA). OPN levels were determined with Enzyme-Linked Immunosorbent Assay by a commercially available assay (Abcam, UK). The intra-assay coefficient of variation was $0.4 \%$ for calcium, $4.0 \%$ for PTH, $4.3 \%$ for $25 \mathrm{OH}-\mathrm{Vitamin}$ D and $<10 \%$ for OPN.

\section{Statistical analysis}

Considering the samples of patients with fractures and without fractures, a $t$ test to compare the levels of OPN which allows to detect an effect size of $80 \%$ of the standard deviation yields a power equal to 0.87 with a significance level of 0.05 . Continuous variables are expressed as mean \pm standard deviation (SD) and categorical variables as percentage. For each variable normality was visually assessed. After normality testing, differences between baseline and follow up were evaluated by paired sample t-test for normally distributed continuous variables and by Mann-Whitney $U$ test for not normally distributed continuous variables. Differences in values for categorical variables between baseline and follow-up were assessed using McNemar's test. The association between OPN at baseline and the presence of fractures at baseline was evaluated by a logistic model. Stepwise elimination using Akaike Information Criterion (AIC) was performed, forcing the model to retain the covariate $25 \mathrm{OH}$-vitamin D and $\mathrm{HbA} 1 \mathrm{c}$ at each iteration. A ROC curve analysis was performed to identify the optimal cutoff of OPN associated with fragility fractures at baseline and the threshold was obtained selecting the highest point in the roc curve according to Younden's Index. The association between $25 \mathrm{OH}$-vitamin D, calcium, PTH and OPN values (covariates) at baseline, and percentage variation of variables (BMI, HbA1c, total cholesterol, HDL-cholesterol, LDL-cholesterol, triglycerides, CKD-EPI, FRAX for major osteoporotic and for hip fracture) between baseline and follow-up, were evaluated with a linear model, after adjusting for age, follow-up duration and basal value of each variable. In the model evaluating the effect of the covariates on the percentage variation of HDL-cholesterol, the results were also adjusted for variation of $\mathrm{HbA1c}$, since glycemic control may influence HDL-cholesterol. A model has been estimated 
for each percentage variation, resulting in 9 models. Stepwise elimination for the covariates was applied using AIC to select the best model. The statistical analysis was performed using the software R package v. 3.3.2. A $p$ value $<0.05$ was considered statistically significant.

\section{Results}

Clinical and biochemical parameters of all patients are reported in Table 1. As shown, no significant differences in clinical and biochemical parameters were found between baseline and follow up. Differences in FRAX scores and CKD-EPI between baseline and follow-up were not significant adjusting for age.

The prevalence of previous fragility fractures was 39.7\% at basal evaluation. Three out of 64 patients reported new fragility fractures (a Colles' fracture, a humeral fracture and a metatarsal fracture, respectively) during the study period. Thus, the fracture incidence was $4.7 \%$ (mean follow-up duration $3.6 \pm 0.9$ years) but the prevalence of fractured patients did not change during the follow-up because the three incident fractures occurred in women already reporting a previous fracture at baseline. No significant differences in clinical and biochemical parameters were found between patients with (n.25) and without (n.39) previous fractures, whereas basal OPN levels were significantly higher in the first group $(0.82 \pm 0.72$ vs $0.36 \pm 0.35 \mathrm{ng} / \mathrm{ml}$, respectively, $p=0.006)$ (Table 2). In the logistic model, the odds of having fractures at baseline increased by $6.7(1.9-31.4,95 \% \mathrm{CI}, p=0.007)$ for each increase of $1 \mathrm{ng} / \mathrm{ml}$ in OPN levels, after adjustment for 25OH-vitamin D levels and HbA1c (Table 3).

A ROC curve analysis was performed to identify the optimal cut-off of OPN associated with fragility fractures at baseline. Using a threshold of $0.33 \mathrm{ng} / \mathrm{ml}$ of OPN a classifier with specificity equal to $72 \%$ and sensitivity equal to $76 \%$ was obtained. The area under the curve was 0.708 (Fig. 1). Clinical and biochemical parameters of patients grouped on the basis of OPN cut-off value are reported in Table 4 . As reported, no significant differences in clinical and biochemical parameters were observed between patients with OPN levels above and below $0.33 \mathrm{ng} / \mathrm{ml}$, except for fracture prevalence.

Higher values of OPN at baseline were also significantly associated with a decrease in HDL-cholesterol levels at follow-up ( $p=0.048$ ), after adjustment for age, basal values of HDL-cholesterol, basal and follow-up values of $\mathrm{HbA1c}$ and duration of follow-up.

Mean serum calcium and PTH levels were $9.6 \pm 0.4 \mathrm{mg} /$ $\mathrm{dl}$ and $49.5 \pm 32.7 \mathrm{pg} / \mathrm{ml}$, respectively, whereas mean
Table 1 Clinical and biochemical parameters

\begin{tabular}{llll}
\hline & Baseline & Follow-up & $p$ Value \\
\hline Age (years) & $67.0 \pm 7.8$ & $70.3 \pm 8.0$ & $0.000^{* * *}$ \\
Diabetes duration (years) & $8.9 \pm 6.7$ & $11.8 \pm 6.3$ & $0.000^{* * *}$ \\
Weight (kg) & $75.6 \pm 12.6$ & $73.4 \pm 14.4$ & 1.0 \\
BMI (kg/m ${ }^{2}$ ) & $30.6 \pm 5.0$ & $30.2 \pm 5.5$ & 0.5 \\
Cardiovascular disease (\%) & 8 & 11 & 0.5 \\
Glycemia (mg/dl) & $145.1 \pm 38.3$ & $150.4 \pm 52.7$ & 0.4 \\
HbA1c (\%) & $7.1 \pm 1.5$ & $7.5 \pm 1.1$ & 0.3 \\
Creatinine (mg/dl) & $0.8 \pm 0.2$ & $0.9 \pm 0.3$ & 0.1 \\
eGFR-CKD EPI (mL/min/1.73 m ${ }^{2}$ ) & $77.2 \pm 17.4$ & $70.4 \pm 23.1$ & $0.5^{\dagger}$ \\
Total cholesterol (mg/dl) & $178.5 \pm 37.1$ & $169.9 \pm 32.4$ & 0.6 \\
HDL-cholesterol (mg/dl) & $51.5 \pm 11.4$ & $52.2 \pm 15.7$ & 0.9 \\
LDL-cholesterol (mg/dl) & $99.9 \pm 33.8$ & $88.2 \pm 29.4$ & 0.8 \\
Triglycerides (mg/dl) & $135.2 \pm 53.4$ & $151.5 \pm 54.2$ & 0.2 \\
FRAX Score (major osteoporotic fractures) & $8.4 \pm 4.7$ & $14.3 \pm 6.1$ & $0.7^{\dagger}$ \\
FRAX score (hip fractures) & $2.8 \pm 2.7$ & $4.3 \pm 3.2$ & $0.6^{\dagger}$ \\
25 OH vitamin D (ng/ml) & $11.4 \pm 5.9$ & - & - \\
Calcium (mg/dl) & $9.6 \pm 0.4$ & - & - \\
PTH (pg/ml) & $49.5 \pm 32.7$ & - & - \\
OPN (ng/ml) & $0.54 \pm 0.57$ & - & - \\
\hline
\end{tabular}

Data are expressed as mean $\pm \mathrm{SD}$ or $\%$

$B M I$ body mass index, $H b A l c$ glycated hemoglobin, $e G F R$ estimated glomerular filtration rate, $C K D E P I$ chronic kidney disease epidemiology collaboration, $H D L$ high density lipoprotein, $L D L$ low density lipoprotein, $P T H$ parathyroid hormone, $O P N$ osteopontin

$* p<0.05 ; * * p<0.01 ; * * * p<0.001 ;{ }^{\dagger}$ adjusted for age 
Table 2 Clinical and biochemical parameters of patients with and without previous fracture

\begin{tabular}{llll}
\hline & Previous fracture (n. 25) & $\begin{array}{l}\text { No previous } \\
\text { fracture (n. 39) }\end{array}$ & $p$ Value \\
\hline Age (years) & $67.2 \pm 8.2$ & $67.0 \pm 7.7$ & 0.9 \\
Diabetes duration (years) & $9.7 \pm 8.6$ & $8.3 \pm 5.1$ & 0.4 \\
Cardiovascular disease (\%) & 8.0 & 7.9 & 0.6 \\
Diabetic neuropathy (\%) & 67 & 68 & 1.0 \\
Diabetic retinopathy (\%) & 7.1 & 13.6 & 0.4 \\
Insulin therapy (\%) & 37 & 45 & 0.4 \\
Weight (kg) & $76.3 \pm 14.9$ & $75.0 \pm 10.9$ & 0.7 \\
BMI (kg/m $\left.{ }^{2}\right)$ & $31.0 \pm 5.6$ & $30.4 \pm 4.6$ & 0.6 \\
HbA1c (\%) & $7.3 \pm 1.6$ & $7.0 \pm 1.4$ & 0.4 \\
eGFR-CKD EPI (mL/min/1.73 m $\left.{ }^{2}\right)$ & $73.2 \pm 18.3$ & $80.0 \pm 16.5$ & 0.2 \\
eGFR-CKD EPI<60 ml/min/1.73 m ${ }^{2}(\%)$ & 33 & 27 & 0.7 \\
Total cholesterol (mg/dl) & $177.2 \pm 31.6$ & $179.3 \pm 40.7$ & 0.8 \\
HDL-cholesterol (mg/dl) & $51.0 \pm 10.1$ & $52.0 \pm 12.3$ & 0.8 \\
LDL-cholesterol (mg/dl) & $100.5 \pm 31.1$ & $99.5 \pm 36.0$ & 0.9 \\
Triglycerides (mg/dl) & $129.0 \pm 38.2$ & $139.6 \pm 62.0$ & 0.4 \\
FRAX score (major osteoporotic fractures) & $9.3 \pm 5.4$ & $7.6 \pm 3.8$ & 0.2 \\
FRAX score (hip fractures) & $3.2 \pm 3.1$ & $2.3 \pm 2.1$ & 0.3 \\
25OH vitamin D (ng/ml) & $11.2 \pm 5.9$ & $11.6 \pm 6.1$ & 0.8 \\
Calcium (mg/dl) & $9.5 \pm 0.4$ & $9.6 \pm 0.4$ & 0.3 \\
PTH (pg/ml) & $47.7 \pm 39.3$ & $50.7 \pm 28.0$ & 0.7 \\
OPN (ng/ml) & $0.82 \pm 0.72$ & $0.36 \pm 0.35$ & $0.006^{* * *}$ \\
\hline
\end{tabular}

Data are expressed as mean $\pm \mathrm{SD}$ or $\%$

$B M I$ body mass index, $H b A l c$ glycated hemoglobin, $e G F R$ estimated glomerular filtration rate, $C K D E P I$ chronic kidney disease epidemiology collaboration, $H D L$ high density lipoprotein, $L D L$ low density lipoprotein, $P T H$ parathyroid hormone, $O P N$ osteopontin

${ }^{*} p<0.05 ; * * p<0.01 ; * * * p<0.001$
Table 3 Logistic regression for prediction of fractures at baseline

\begin{tabular}{lll}
\hline Parameter & $p$ & Odds ratio $(95 \%$ CI) \\
\hline 25OH-vitamin D & 0.490 & $0.9(0.8-1.0)$ \\
HbA1c & 0.184 & $1.3(0.8-2.0)$ \\
OPN & $0.007^{* *}$ & $6.7(1.9-31.4)^{* *}$ \\
\hline
\end{tabular}

$H b A 1 c$ glycated hemoglobin, $O P N$ osteopontin

$* p<0.05 ; * * p<0.01 ; * * * p<0.001$

levels of $25 \mathrm{OH}$-vitamin D were insufficient in the study population $(11.4 \pm 5.9 \mathrm{ng} / \mathrm{ml})$. None of the patients had received calcium and vitamin $D$ supplementation at baseline and in the previous 6 months from baseline. In the longitudinal evaluation, after adjustment for age, of $25 \mathrm{OH}$-vitamin D levels and time of observation, basal $25 \mathrm{OH}$-vitamin-D values were significantly $(p=0.029)$ associated with an increase in the FRAX-estimated probability of hip fracture at follow-up visit.

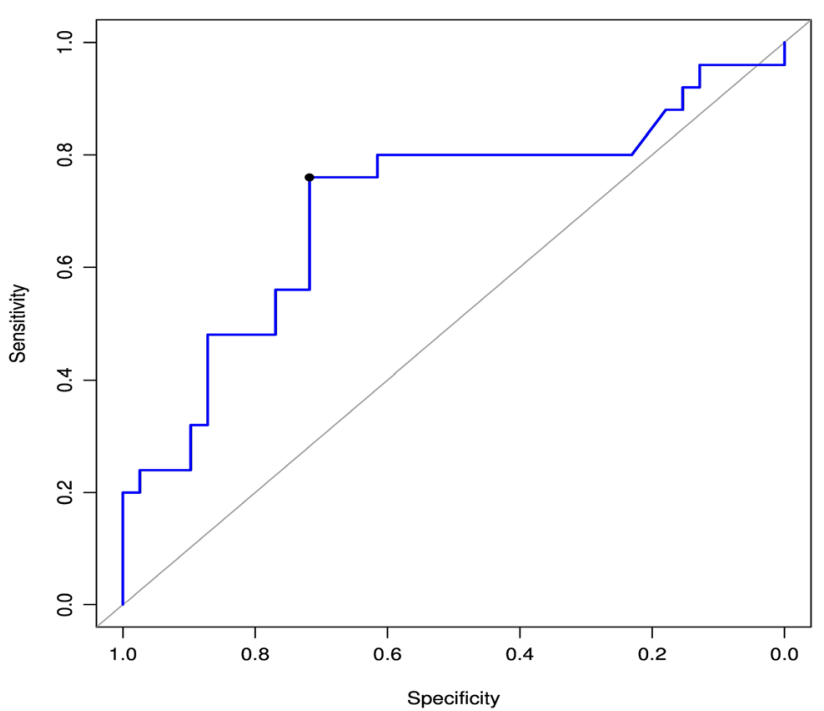

Fig. 1 ROC curve for OPN and previous fractures at baseline. Using a threshold of $0.33 \mathrm{ng} / \mathrm{ml}$ of OPN a classifier with specificity equal to $72 \%$ and sensitivity equal to $76 \%$ was obtained. The area under the curve was 0.708 
Table 4 Clinical and biochemical parameters of patients grouped by ROC curve OPN cut-off

\begin{tabular}{llll}
\hline & $\begin{array}{l}\text { OPN } \\
\leq 0.33 \mathrm{ng} / \mathrm{ml} \\
(\mathrm{n} .34)\end{array}$ & $\begin{array}{l}\text { OPN } \\
>0.33 \mathrm{ng} / \mathrm{ml} \\
(\mathrm{n} .30)\end{array}$ & $p$ Value \\
\hline Age (yeas) & $65.6 \pm 7.9$ & $68.6 \pm 7.5$ & 0.2 \\
Diabetes duration (years) & $7.9 \pm 4.1$ & $10.1 \pm 8.7$ & 0.9 \\
Cardiovascular disease (\%) & 5.8 & 10.0 & 0.8 \\
Diabetic neuropathy (\%) & 59 & 53.3 & 0.5 \\
Diabetic retinopathy (\%) & 17.6 & 10 & 0.4 \\
BMI (kg/m ${ }^{2}$ ) & $30.6 \pm 5.0$ & $30.6 \pm 5.2$ & 1.0 \\
HbA1c (\%) & $7.2 \pm 1.3$ & $7.1 \pm 1.7$ & 0.4 \\
eGFR-CKD EPI <60 ml/ & 12 & 20 & 0.7 \\
min/1.73 m ${ }^{2}(\%)$ & & & \\
Total cholesterol (mg/dl) & $180.3 \pm 38.0$ & $176.2 \pm 36.8$ & 0.7 \\
HDL-cholesterol (mg/dl) & $52.1 \pm 12.3$ & $51.0 \pm 10.5$ & 0.6 \\
LDL-cholesterol (mg/dl) & $100.3 \pm 32.0$ & $99.4 \pm 36.7$ & 0.9 \\
Triglycerides (mg/dl) & $140.0 \pm 60.0$ & $129.5 \pm 45.3$ & 0.4 \\
Previous fractures (\%) & 17.6 & 63.3 & $0.000^{* * * *}$ \\
Incident fractures n. & 0 & 3 & 0.06 \\
\hline
\end{tabular}

Data are expressed as mean $\pm \mathrm{SD}$ or as frequency

$B M I$ body mass index, $H b A 1 c$ glycated hemoglobin, $e G F R$ estimated glomerular filtration rate, $C K D E P I$ chronic kidney disease epidemiology collaboration, $H D L$ high density lipoprotein, $L D L$ low density lipoprotein

${ }^{*} p<0.05 ; * * p<0.01 ; * * * p<0.001$

\section{Discussion}

In this study, levels of OPN were higher in post-menopausal women with T2DM with prevalent fractures than in patients without clinical fractures. The increased fracture risk despite normal or even higher BMD values is a pathogenetic unresolved paradox in patients with T2DM [15]. Such a lower reliability of BMD has been related to alteration in bone quality [15]. Moreover, non-skeletal factors could also heavily influence fracture risk $[1,15]$. The clinical challenge of fracture risk estimate in T2DM patients is further compounded by the generally low predictive performance of FRAX, the most commonly used algorithm based on clinical factors $[7,8]$. The results of our previous crosssectional multicenter study confirmed that patients with T2DM had higher fracture rate than control subjects, despite equal or even lower FRAX scores [8]. Given this scenario, any new useful tool to investigate fracture risk in T2DM patients could add to the current clinical practice. Several biochemical markers of bone metabolism have been shown to be reduced in T2DM, reflecting a low turnover rate, and are not useful to predict fracture risk in these patients [16]. The possible role of OPN on bone health is supported by several basic science and even clinical data. OPN seems to promote osteoclast function [9] but compared to the effect on bone resorption, much less is known about the role of OPN in bone formation. Interestingly, it has been observed that it might act as a negative regulator of pre-osteoblastic cell proliferation and differentiation in vitro [10]. At a hypothetical level, the inhibitory effect of high OPN levels on osteoblasts might indirectly have a negative influence on osteoclast function. Thus, high OPN levels might be linked to low bone resorption and formation, in line with the low bone turnover pattern, observed in T2DM. However, a limit of this study is the lack of the determination of bone resorption and apposition markers. Further studies are needed to investigate this possible link between high OPN levels and low bone turnover in T2DM. Moreover, in clinical grounds, previous studies have shown a negative association between OPN and BMD in post-menopausal women [17, 18]. Since the number of incident fractures during the follow-up period was quite low $(n=3)$, we could not reasonably test the association between OPN levels and incident fractures. Although larger studies with longer follow-up duration are needed to confirm these findings, OPN measurement might be a useful marker of fracture risk in clinical practice.

As additional information, our data also showed an association between OPN and HDL-cholesterol. Even though we did not find any significant correlation with hard end-points, as myocardial infarction or stroke, this information has some speculative interest. It should be stressed that the significant association between OPN and HDL-cholesterol was confirmed after adjustment for HbA1c levels, since HDL-cholesterol values are influenced by the degree of glycemic control [19]. This finding is relevant, because post-menopausal women are more prone to dyslipidemia and to increased cardiovascular mortality [20,21], and the link between low HDL-cholesterol levels and increased coronary heart disease (CHD) morbidity and mortality is supported by consistent evidence [22, 23]. On the other hand, serum OPN has been reported to be increased in patients with atherosclerosis and myocardial infarction [23]. OPN can induce macrophage chemotaxis [24]. Thus, it may play an important role in several chronic inflammatory conditions, including atherosclerosis. In particular, it has been shown that circulating OPN might be a marker of early coronary vascular calcification in T2DM patients with asymptomatic coronary artery disease [25]. These findings seem to support the role of OPN as an emerging biomarker of cardiovascular risk in T2DM [12]. In line with our results, in a recent study, OPN levels were also found to be correlated to lower HDL-cholesterol in patients with type 1 diabetes [26]. Thus, circulating levels of OPN could be predictive of an unfavourable lipid profile and, consequently, high cardiovascular risk in patients with T2DM.

Moreover, the results of this study show that, despite no significant difference in other parameters was found, low levels of $25 \mathrm{OH}$-vitamin D were associated with a subsequent increase of FRAX-estimated fracture risk. Even if the FRAX 
algorithm was not set-up for the longitudinal monitoring of fracture risk, this result deserves some interest. In fact, it persisted after adjustment for age, and was largely due to both incident hip fractures in parents and to the incident fractures occurred in T2DM patients during the follow-up. Although firm conclusions cannot be drawn from these data, we speculate that the poor vitamin D status of these patients associates to a worsening estimated fracture risk profile in T2DM. If confirmed in larger series, our results could suggest to integrate FRAX algorithm with vitamin D status in T2DM patients.

The limits of this study are the small sample size, the lack of BMD-based FRAX assessment and of systemic inflammation markers. However, the results are intriguing for future studies in this field.

\section{Conclusions}

In conclusion, in post-menopausal women with T2DM, OPN levels relate to prevalent fractures and to HDL-cholesterol changes over time, which could reflect a role of OPN in hypothetical pathways shared by different diabetic chronic conditions.

Acknowledgements This research did not receive any specific grant from funding agencies in the public, commercial, or no-for- 26 profit sectors.

\section{Compliance with ethical standards}

Conflict of interest The authors declare that they have no conflict of interest.

Ethical approval All procedures performed in studies involving human participants were in accordance with the ethical standards of the institutional and/or national research committee and with the 1964 Helsinki declaration and its later amendments or comparable ethical standards. This article does not contain any studies with animals performed by any of the authors.

Informed consent Informed consent was obtained from all individual participants included in the study.

\section{References}

1. Carnevale V, Romagnoli E, D’Erasmo E (2004) Skeletal involvement in patients with diabetes mellitus. Diabetes Metab Res Rev 20(3):196-204

2. Schwartz AV, Vittinghoff E, Bauer DC, Hillier TA, Strotmeyer ES, Ensrud KE, Study of Osteoporotic Fractures (SOF), the Osteoporotic Fractures in Men (MrOS), Health, Aging and Body Composition (Health ABC) et al (2011) Association of BMD and FRAX score with risk of fracture in older adults with type 2 diabetes. JAMA 305(21):2184-2192. https://doi.org/10.1001/ jama.2011.715
3. Vestergaard P (2007) Discrepancies in bone mineral density and fracture risk in patients with type 1 and type 2 diabetesa meta-analysis. Osteoporosis Int 18(4):427-444. https://doi. org/10.1007/s00198-006-0253-4

4. Janghorbani M, Van Dam RM, Willett WC, Hu FB (2007) Systematic review of type 1 and type 2 diabetes mellitus and risk of fracture. Am J Epidemiol 166(5):495-505. https://doi. org/10.1093/aje/kwm106

5. Fan Y, Wei F, Lang Y, Liu Y (2016) Diabetes mellitus and risk of hip fractures: a meta-analysis. Osteoporos Int 27(1):219-228. https://doi.org/10.1007/s00198-015-3279-7

6. Centre for Metabolic Bone Diseases. University of Sheffield UK. https://www.sheffield.ac.uk/FRAX/tool.jsp?lang=it. Accessed Jun 2016

7. Giangregorio LM (2012) FRAX underestimates fracture risk in patients with diabetes. J Bone Miner Res 27:301-308

8. Carnevale V, Morano S, Fontana A, Annese MA, Fallarino M, Filardi T et al (2014) Assessment of fracture risk by the FRAX algorithm in men and women with and without type 2 diabetes mellitus: a cross-sectional study. Diabetes Metab Res Rev 30:313-322

9. Chellaiah MA, Kizer N, Biswas R, Alvarez U, Strauss-Schoenberger J, Rifas L et al (2003) Osteopontin deficiency produces osteoclast dysfunction due to reduced CD44 surface expression. Mol Biol Cell 14(1):173-189

10. Huang W, Carlsen B, Rudkin G, Berry M, Ishida K, Yamaguchi DT et al (2004) Osteopontin is a negative regulator of proliferation and differentiation in MC3T3-E1 pre-osteoblastic cells. Bone 34:799-808

11. Mazzali M, Kipari T, Ophascharoensuk V, Wesson JA, JohnsonR Hughes J (2002) Osteopontin-A molecule for all seasons. QJM 95:3-13

12. van der Leeuw J, Beulens JW, van Dieren S, Schalkwijk CG, Glatz JF, Hofker MH et al (2016) Novel biomarkers to improve the prediction of cardiovascular event risk in type 2 diabetes mellitus. J Am Heart Assoc 5:e003048. https://doi.org/10.1161/ JAHA. 115.003048

13. Hanwell HE, Vieth R, Cole DE, Scillitani A, Modoni S, Frusciante V, Ritrovato G, Chiodini I, Minisola S, Carnevale V (2010) Sun exposure questionnaire predicts circulating 25-hydroxyvitamin D concentrations in Caucasian hospital workers in southern Italy. J Steroid Biochem Mol Biol 121(1-2):334-337. https:// doi.org/10.1016/j.jsbmb.2010.03.023 (epub 2010 Mar 16)

14. Levey AS, Stevens LA, Schmid CH, Zhang YL, Castro AF 3rd, Feldman HI et al (2009) A new equation to estimate glomerular filtration rate. Ann Intern Med 150(9):604-612

15. Carnevale V, Romagnoli E, D’Erasmo L, D'Erasmo E (2014) Bone damage in type 2 diabetes mellitus. Nutr Metab Cardiovasc Dis 24:1151-1157

16. Dobnig H, Piswanger-Solkner JC, Roth M, Obermayer-Pietsch B, Tiran A, Strele A et al (2006) Type 2 diabetes mellitus in nursing home patients: effects on bone turnover, bone mass, and fracture risk. J Clin Endocrinol Metab 91:3355-3363

17. Cho EH, Cho KH, Lee HA, Kim SW (2013) High serum osteopontin levels are associated with low bone mineral density in postmenopausal women. J Korean Med Sci 28(10):1496-1499. https://doi.org/10.3346/jkms.2013.28.10.1496 (epub 2013 Sep 25)

18. Fodor D, Bondor C, Albu A, Simon SP, Craciun A, Muntean L (2013) The value of osteopontin in the assessment of bone mineral density status in postmenopausal women. J Investig Med 61(1):15-21. https://doi.org/10.2310/jim.0b013e3182761264

19. Barbarossa G, Renzi A, D'Erasmo L, Gallo A, Grieco E, Rossetti $M$ et al (2014) The relation between glycemic control and HDL-C in type 2 diabetes: a preliminary step forward? Diabetes Res Clin Pract 104:e26-e28 
20. Nakhjavani M, Morteza A, Esteghamati A, Khalilzadeh O, Zandieh A, Safari R (2011) Serum lipoprotein(a) levels are greater in female than male patients with type 2 diabetes. Lipids 46:349-356

21. Kautzky-Willer A, Kamyar MR, Gerhat D, Handisurya A, Stemer G, Hudson S et al (2010) Sex-specific differences in metabolic control, cardiovascular risk, and interventions in patients with type 2 diabetes mellitus. Gend Med 7:571-583

22. Wilson PWF, D'Agostino RB, Levy D, Belanger AM, Silbershatz H, Kannel WB (1998) Prediction of coronary heart disease using risk factor categories. Circulation 97:1837-1847

23. Tamura A, Shingai M, Aso N, Hazuku T, Nasu M (2003) Osteopontin is released from the heart into the coronary circulation in patients with a previous anterior wall myocardial infarction. Circ J 67:742-744

24. Gravallese EM (2003) Osteopontin: a bridge between bone and the immune system. J Clin Invest 112:147-149

25. Berezin AE, Kremzer AA (2013) Circulating osteopontin as a marker of early coronary vascular calcification in type two diabetes mellitus patients with known asymptomatic coronary artery disease. Atherosclerosis 229:475-481

26. Barchetta I, Alessandri C, Bertoccini L, Cimini FA, Taverniti L, Di Franco $M$ et al (2016) Increased circulating osteopontin levels in adult patients with type 1 diabetes mellitus and association with dysmetabolic profile. Eur J Endocrinol 174:187-192 